Community Health Sheffield for their participation in the project.

original papers

\section{References}

BLUMENTHAL, S. \& WESSELY, S. (1994) The cost of Mental Health Review Tribunals. Psychiatric Bulletin, 18, $274-276$.

DAVISON, P. \& PEREZ DE ALBENIZ, A (1997) Reports prepared for mental health review tribunals and managers' reviews. Psychiatric Bulletin, 21 $364-366$
REGIONAL CHAIRMEN OF THE MENTAL HEALTH REVIEW TRIBUNAL FOR ENGLAND AND WALES (2000) Guidance for the Preparation of Medical Reports for the MHRT. London: Mental Health ReviewTribunal Secretariat. (1998) Mental health review tribunal medical reports. Psychiatric Bulletin, 22, 615-618.

LANGLEY, G. E. (1993) Mental health review tribunals in practice. Psychiatric Bulletin, 17, 331-336.

ROSS HAMILTON, PETER GORDON AND SIMON NAJI

\title{
Service innovation: the first year of a brief psychiatric screening clinic in primary care
}

\section{AIMS AND METHOD}

To introduce a monthly screening clinic for new patients referred to the community mental health team with less severe mental health problems.

\section{RESULTS}

Sixty patients were selected for screening in the first year. Their non-attendance rate of $48 \%$ was more than double the rate for all new patients. We did not diagnose severe mental illness in any patients on first assessment or during the 6 months of follow-up.

\section{CLINICAL IMPLICATIONS}

Patients referred from general practice with minor psychiatric morbidity may have particularly high rates of non-attendance. The brief screening clinic model offered us considerable savings in consulting time. The outcome for our service is shorter waiting times for patients with more severe mental health problems.
General adult psychiatrists report increasing case-loads (Mynors-Wallis, 2001) and plummeting morale (Deahl \& Turner, 1997). The case-loads of consultants in community mental health teams (CMHTs) have been described recently as too large for responsible medical officers to exercise their statutory duties (Tyrer et al, 2001). In addition, the number of new referrals to psychiatric outpatient clinics reportedly increased by $13 \%$ in England and Wales between 1987-1988 and 1997-1998 (Harvey et al, 2000), with $38 \%$ more recorded non-attendance in that time period.

There is evidence that referral decisions dictating pathways to care for people with mental health problems are not based upon the diagnosed mental health problem (Morgan, 1989). Furthermore, there is recognition of the growing tension at the interface between mental health services and primary care as to roles and referral criteria (Gask et al, 1997). A recent survey of more than 200 mental health professionals (psychiatrists, general practitioners (GPs) and psychologists) (Ogden \& Pinder, 1997) implies that referral guidelines may in fact be detrimental. No consensus among professionals could be reached in this study as to who was the appropriate practitioner to deal with the vast majority of mental health problems.
From this backdrop, and in the face of annual increments in new referrals to our own CHMT, we sought to introduce a brief screening clinic for selected referrals from GPs to our service. The concept of triage in emergency psychiatry has recently been described (Morrison et al, 2000), suggesting that it is an effective method of introducing flexibility of response and encouraging continuity of patient care. We have previously described our own views on pathways to care for out-of-hours psychiatric referrals (Gordon \& Hamilton, 1997). However, the following study concentrates on 'triage' within routine referrals to a CMHT.

\section{The study}

Our CMHT accepts referrals from all GPs in the towns of Peterhead and Fraserburgh in Aberdeenshire (population 37653). Those towns are recognised as the most deprived areas of Grampian (McLoone, 2000).

From 1 June 1999 until 31 May 2000, all new patient referrals from GPs to the team were considered for allocation to the screening clinics. At the referral allocation meeting, and using the GP's referral letter and any previous psychiatric case records, an attempt was made to identify patients from the general pool of referrals 
who had no markers of severe enduring mental illness. Thus, patients were selected for the screening clinic when there was no documented past psychiatric history and usually when the GP had diagnosed mild depression, adjustment disorder or neuroses.

The screening clinics were held on one afternoon per month in the same venues as the 'shifted out-patient clinics' (Tyrer, 1984). These clinics are run on a weekly basis in the two general practices and cottage hospitals within the towns. Between four and six of the selected patients were invited to attend the monthly screening clinics. A 20-minute appointment was offered with either a consultant psychiatrist or specialist registrar, accompanied by one other member of the CMHT, usually a community psychiatric nurse, psychiatric social worker or occupational therapist. At the time of this initial assessment any patient judged to have a severe mental illness (see Table 1) was to be offered a full psychiatric evaluation at the next available clinic (usually within 7 days). Alternatively, of course, patients could be admitted to hospital from the clinic for further assessment.

\section{Aims of the project}

We had previously observed that many patients referred with minor psychiatric morbidity did not attend for the initial appointment. We postulated that such nonattendance may be owing to resolution of some personal or social crisis within the period between referral and the offer of the first assessment appointment (on average about 4-6 weeks later). In addition, we had also noted that many patients with less severe psychiatric problems were discharged after first assessment. The 6 -month follow-up data were collected to allow us to examine outcome for clinic non-attenders and patients discharged at their first visit. Scrutiny of the diagnoses on first assessment at the screening clinic enabled us to gauge our accuracy in selecting patients for screening who appeared to have less severe problems from their referral letters.

By screening up to six new referrals at 20-minute intervals in the brief clinic there was the potential to free up 4 hours of assessment time given that our traditional model was to offer a 1-hour slot for each new referral. There appeared then the opportunity to shorten waiting times for first assessments for patients with more severe mental health problems. The screening clinic also held potential to avoid clinicians being 'stranded' in peripheral settings with unfilled new patient slots and no identifiable clinical work to occupy that time. We also hoped that joint assessment procedures within the team would be mutually beneficial in terms of team building and education.

It was recognised that medical staff would perform the assessment interview but that other members of the team may have more extensive knowledge of local services for patients with social or personal difficulties.

\section{Method}

A thorough case note review (to include a 6 -month follow-up period after first appointment) was undertaken for all 60 patients referred to the screening clinic in the 12 -month study period. For patients who attended, an International Classification of Diseases (ICD-10, World Health Organization, 1992) opening diagnosis was available in all cases from the episode opening documentation returned to the medical records department at the time of the first assessment. Scrutiny of those attenders' records for the following 6 months allowed us to examine whether such patients continued treatment with the CMHT. If the patient was discharged at first assessment then notes were reviewed to establish whether they were referred back to the CMHT. We also checked for evidence of referral to the liaison psychiatric services following episodes of deliberate self-harm and whether there had been a psychiatric hospital admission or rereferral to any of the psychiatry sub-specialities. We also reviewed the return data to our trust from the Registrar of Deaths, which includes all suicides and suspicious deaths. Similarly for non-attenders we reviewed all psychiatric case notes for re-referral, or contact with any of the other services noted above, in the 6-month period following the missed appointment.

\section{Findings}

During the 12 months of the study, 60 patients were selected from a total of 334 referrals to the allocation meetings. This represents around $18 \%$ of new referrals and all 60 were invited to the screening clinics. The ratio of males to females was 26:34 and only five patients were over 50 years of age. Twenty-nine (48\%) did not attend their first appointment, more than double the $21 \%$ non-attendance rate for the same time period for all 334 new referrals to the team.

The diagnosis on episode opening from the 31 patients who attended for first assessment is recorded in Table 1. The most frequent episode opening diagnoses are adjustment disorder $(n=9 ; 29 \%)$ and nil psychiatric $(n=5$; $16 \%$ ). The diagnoses under the heading 'other' were posttraumatic stress disorder, dysmorphophobia, generalised anxiety disorder, two adverse events from childhood and one other personality disorder. Substance misuse, depressive episodes, either mild or in remission, and other consequences of childhood events make up the remainder of opening diagnoses. None of the patients who attended for initial assessment were diagnosed to have a psychotic illness or moderate or severe depression. No patients were admitted from the screening clinic or recalled for urgent review.

The 6-month follow-up data are presented for clinic attenders and non-attenders (Table 2). From Table 2 just over a quarter $(n=8 ; 26 \%)$ of the 31 attenders were taken on for further treatment with the CMHT. The majority was discharged after first assessment. From those 23 people discharged at first assessment, 19 (83\%) had no further secondary care contact in the 6 -month follow-up period. Of the four people referred back to original papers 
Table 1. ICD-10 opening diagnosis on screening (World Health Organization, 1992)

original

papers

Clinic attenders $(n=31)$
Diagnosis

$n(\%)$

Adjustment disorder

9 (29)

Other diagnoses

6 (19)

Nil psychiatric

$5(16)$

Substance/alcohol misuse or dependency

$4(13)$

Problems relating to alleged childhood abuse

$3(10)$

Mild depressive episode

$2(6)$

Recurrent depression currently in remission

$2(6)$

Table 2. Six-month follow-up for clinic attenders and non-attenders

Outcome

$n(\%)$

For attenders $(n=31)$

Taken on by CMHT

Admitted from first assessment or recalled

for urgent review

$0(0)$

Discharged at time of first assessment

For non-attenders $(n=29)$

No further contact with mental health services

Referred back to mental health services

$\mathrm{CMHT}$

$3(10)$

Liaison clinic

1 (3)

Substance misuse

$1(3)$

CMHT, community mental health team.

mental health services by their GP in the follow-up period, one was referred to the substance misuse service, one each to clinical psychology and psychotherapy and one was referred back to the CMHT. The patient referred back to us was discharged again after first assessment with a diagnosis of anxiety.

From those 29 patients from Table 2 who did not attend, $24(83 \%)$ were not referred back to mental health services in the follow-up period. Of the five patients who were referred, three $(10 \%)$ were rereferred again to the CMHT (all three were seen once and discharged from CMHT follow-up at first assessment). Two further patients from the non-attenders at our clinic had subspeciality referrals, one at the liaison psychiatry clinic (this patient was discharged at first assessment with diagnosis of anxiety) and another at the substance misuse clinic (diagnosis of opiate dependency).

None of the patients in either group were admitted to hospital, seen by the liaison psychiatrists after deliberate self-harm or notified subsequently as a suspicious death or suicide.

\section{Discussion}

In simple terms, considering only medical staff hours, the 29 patients who did not attend used only 9 hours 40 minutes of consulting time in comparison to 29 hours in the traditional clinic; a saving of 19 hours and 20 minutes.
The 31 attenders were assessed in 10 hours 20 minutes as opposed to 31 hours, a further saving of 20 hours 40 minutes. In the year of the study this represented more than 40 hours of saved consulting time equivalent to more than six all day clinics for one member of medical staff, bearing in mind that at least 2 hours of the working day is spent travelling to these rural clinics.

We were heartened by our apparent ability to invite to this clinic only those patients with minor morbidity, as judged from the diagnoses of those who attended. In other words, none of our attenders appeared to have a major mental illness. Probably more crucially, given the high non-attendance rate of almost 50\%, we did not find evidence of significant numbers from that group being referred back to psychiatric services, admitted to hospital or assessed after self-harming in the 6 months after their non-attendance.

Finally, we accept that some colleagues may find the move away from a detailed traditional first psychiatric assessment interview heretical. We feel that the evidence cited in the introduction with regard to rising case-loads and primary-secondary care interface difficulties calls for imaginative solutions. Our data may lead some observers to question whether many of our screening clinic patients should have been referred at all. However, we foresee that GPs will continue to envisage different referral priorities to the CMHT staff in spite of close geographical and personal working relationships and the screening clinic represents one potential interface solution.

\section{The future}

First, we recognise limitations of our study. Ideally we would have liked to canvas opinions of referrers to the clinic and also the patients' view of the assessment. The time period of follow-up of 6 months is relatively short, and, although we reviewed every set of psychiatric records in detail, a more complete picture of outcome would have been available from including GP case note review or even interviewing the patients. This first study represents a feasibility project within a busy $\mathrm{CMHT}$ and the screening clinics are now running in two local mental health teams with a third team preparing to pilot their own project. The next stage for our own team is to seek funding for a more rigorous prospective assessment of the clinics.

\section{Acknowledgements}

We are grateful to Joaane Coull and Morag Williamson for secretarial support and to Pat Grant at the Medical Records Department, Royal Cornhill Hospital.

\section{Declaration of interest}

None. 


\section{References}

DEAHL, M. \& TURNER, T. (1997) General psychiatry in no-man's land. British Journal of Psychiatry, 171 6-8.

GASK, L., SIBBALD, B. \& CREED, F. (1997) Evaluating models of working at the interface between mental health services and primary care. British Journal of Psychiatry, 170 6-11.

GORDON, P. J. \& HAMILTON, R. J. (1997) Psychiatric self referral in Scotland - an unknown quantity? Health Bulletin, $\mathbf{5 5}$ 237-242.
HARVEY, N. S., KOHEN, D. \& HOLLANDER, D. (2000) The evolution of the psychiatric out-patient clinic Primary Care Psychiatry, 6, 37.

McLOONE, P. (2000) Carstairs Scores for Scottish Postcode Sectors from the 1991 Census. Glasgow: Public Health Research Unit, University of Glasgow.

MORGAN, D. (1989) Psychiatric cases an ethnography of the referral process. Psychological Medicine, 19, 243-253.
MORRISON, A., HULL, A. \&

SHEPHARD, B. (2000) Triage in

emergency psychiatry. Psychiatric

Bulletin, 24, 261-264.

MYNORS-WALLIS, L. (2001)

Case-loads to workloads - the role

of the general adult psychiatrist.

Psychiatric Bulletin, 25, 1-2.

OGDEN, J. \& PINDER, K. (1997)

Mental health referrals: a matter for consensual quidelines? Primary Care

Psychiatry, 3, 31-36.

TYRER, P. (1984) Psychiatric clinics in general practice. An extension of community care. British Journal of Psychiatry, 145, 9-14.

- AL MUDERIS, O. \& GULBRANDSEN

D. (2001) Distribution of case-load in

community mental health teams.

Psychiatric Bulletin, 25, 10-12.

WORLD HEALTH ORGANIZATION

(1992) The ICD-10 Classification of Mental and Behavioural Disorders.

Geneva: WHO.

*Ross Hamilton Senior Clinical Research Fellow, Peter Gordon Specialist Registrar, Royal Cornhill Hospital, Cornhill Road, Aberdeen AB25 2ZH, Simon Naji Programme Director, Health Services Research Unit, University of Aberdeen 